(C) [2002] IEEE. Reprinted, with permission, from [ Kodagoda R. S. Kodagoda, Wijerupage S. Wijesoma and Arjuna P. Balasuriya, Road Curb and Intersection Detection Using A 2D LMS , Proc. of the IEEE/RSJ Int. Conf. on Intelligent Robots and Systems (IROS 2002)]. This material is posted here with permission of the IEEE. Such permission of the IEEE does not in any way imply IEEE endorsement of any of the University of Technology, Sydney's products or services. Internal or personal use of this material is permitted. However, permission to reprint/republish this material for advertising or promotional purposes or for creating new collective works for resale or redistribution must be obtained from the IEEE by writing to pubspermissions@ieee.org. By choosing to view this document, you agree to all provisions of the copyright laws protecting it 


\title{
Road Curb and Intersection Detection Using A 2D LMS
}

\author{
Kodagoda R. S. Kodagoda, Wijerupage S. Wijesoma and Arjuna P. Balasuriya \\ School of Electrical and Electronic Engineering, \\ Nanyang Technological University, Singapore \\ e-mail: eswwijesoma@ntu.edu.sg
}

\begin{abstract}
In most urban roads, and similar environments such as in theme parks, campus sites, industrial estates, science parks and the like, the painted lane markings that exist may not be easily discernible by CCD cameras due to poor lighting, bad weather conditions, and inadequate maintenance. An important feature of roads in such environments is the existence of pavements or curbs on either side defining the road boundaries. These curbs, which are mostly parallel to the road, can be harnessed to extract useful features of the road for implementing autonomous navigation or driver assistance systems. However, extraction of the curb or road edge feature using vision image data is a very formidable task as the curb is not conspicuous in the vision image. To extract the curb using vision data requires extensive image processing, heuristics and very favorable ambient lighting. In our approach, road curbs are extracted speedily using range data provided by a $2 D$ Laser range Measurement System (LMS). Experimental results are presented to demonstrate the viability, and effectiveness, of the proposed methodology and its robustness to different road configurations including road intersections.
\end{abstract}

\section{Introduction}

Camera alone methodologies for road detection $[1,2]$ are extensively favored, researched and tested by the research community. Millimeter Wave Radars (MMWR) also being used in few instance for road and obstacle detection [3]. In the past decade, interests in the use of laser range measurement systems in autonomous navigation systems have been on the increase [4, 6-8]. A laser range measurement system can provide a low cost alternative to MMWR systems for obstacle detection and depth/range measurement under poor lighting, visibility and bad weather conditions [5]. In autonomous navigation, laser measurement systems have been used in obstacle detection [4, 6], navigation [7] and in some cases localization [8]. In our application, the laser measurement system is used mainly to detect the road boundaries or edges as defined by the curbs. Use of laser measurement device to detect curbs is more robust and reliable as compared to camera-based vision as Road curbs unlike painted lane markings are not conspicuous and hence not easily extracted from a camera image.

In section 2 data segmentation algorithm using Extended Kalman Filtering (EKF) is described. Feature identification and extraction is detailed in section 3. Section 4 concludes the paper.

\section{Filtering and segmentation}

The SICK planar two-dimensional (2D) laser measurement system (LMS) [5] is rigidly affixed to the vehicle frame as shown in Figure 1. To obtain the road edge or curb information, it may be noted that the LMS unit is mounted looking down the road ahead with zero pan and swing angles and a small tilt angle. The LMS scans a laser spot beam from right to left on a plane inclined at an angle $\alpha_{L}$ (tilt angle) to the road surface. It is assumed that the road is flat and horizontal. The road curb surfaces at both sides are assumed vertical and the immediate pavement surfaces are assumed horizontal and flat (Figure 2). Under the above assumptions, a single planar sweep of the laser spot beam yields different sets of range data points corresponding to the pavement surfaces on either side of the road ( $A B$ and $E F$ of Figure 2), road edge surfaces ( $B C$ and $D E$ of Figure 2), and the road surface ( $C D$ of Figure 2). Each of the set of points, under ideal and assumed conditions, defines a straight-line segment since the intersection of two planes (laser scanning plane and the external surface plane) gives rise to a straight line. Thus, it may be noted that the road edges corresponding to points of intersection of the line segments or the points at which there is significant discontinuity or change of gradient of the path trace of the laser beam. In practice measurement noise and other sources of noise (specula reflections) can corrupt the data points thus making it difficult to detect the 
points of discontinuity. The problem of noise filtering and simultaneous detection of the points of discontinuity (or segmentation of data into edges or lines) are handled by an extended Kalman filter. For a given region (e.g. road surface, curb surface or pavement surface) the evolution of the range data, $\left(d_{i}\right)$, provided by the LMS can be described approximately by a straight-line over a small window. This straightline process model is used to predict the next range data $\left(d_{i+2}\right)$ given the past two measurements $\left(d_{i}, d_{i+1}\right)$. The filtering is effective and valid provided the model of the process adequately describes the evolution of the range data points. This is valid for data lying on the same region (pavement surface, vertical curb surface and road surface). The prediction error would be significant from the measured data at the boundary separating two contiguous regions (horizontal pavement surface and vertical curb surface), since the process model parameters for the two regions are different, or in other words line segments will not overlap for the two regions. Thus, the magnitude of the prediction error computed at a particular data point can be used to validate the authenticity of the process model describing the evolution of the range data points. If the prediction error exceeds a threshold at a particular data point it indicates the start of a new process model, and hence a candidate edge point.

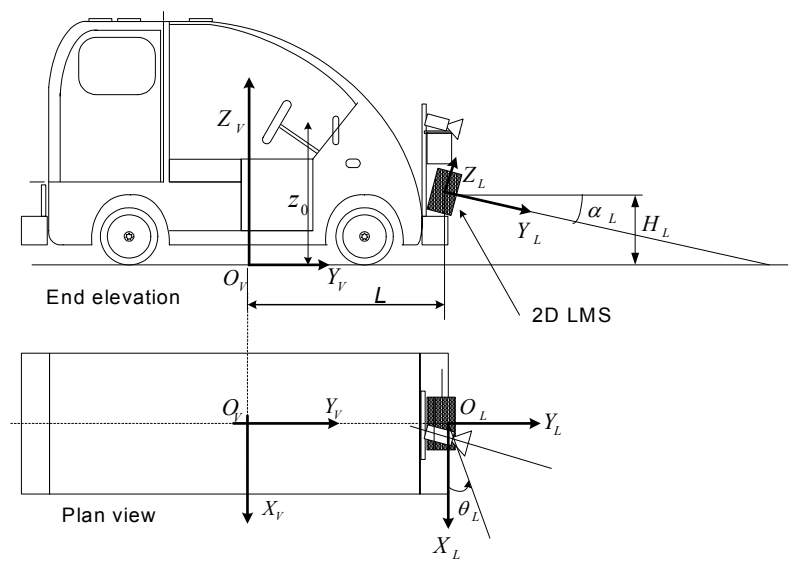

Figure 1: Coordinate systems

\subsection{EKF for edge segmentation}

Consider the three points $\mathrm{P} 1, \mathrm{P} 2$ and $\mathrm{P} 3$, lying on a line segment $\mathrm{L} 1$, at a range of $d_{i}, d_{i+1}$ and $d_{i+2}$ respectively from the laser measuring system, as shown in Figure 3. Using elementary trigonometry it can be shown that:

$$
d_{i+2}=\frac{d_{i} d_{i+1}}{2 d_{i} \cos \gamma-d_{i+1}}
$$

where, $\gamma$ is the angular separation between two laser range measurements. Now, we setup up the process model by choosing the state variables $x_{1}$ and $x_{2}$ as follows:

$$
\begin{aligned}
& x_{1}(k+1)=d_{i+2} \\
& x_{2}(k+1)=x_{1}(k)=d_{i+1}
\end{aligned}
$$

$x_{1}(k)$ and $x_{2}(k)$ represent the states at the pseudo discrete time index $k$. Hence from equations (1) and (2) we obtain the second order non-linear process model:

$$
\begin{aligned}
& x_{1}(k+1)=\frac{x_{2}(k) x_{1}(k)}{2 x_{2}(k) \cos \gamma-x_{1}(k)}+w_{1}(k+1) \\
& x_{2}(k+1)=x_{1}(k)+w_{2}(k+1)
\end{aligned}
$$

That is, in vector form:

$$
\mathbf{x}(k+1)=\mathbf{F}(\mathbf{x}(k))+\mathbf{w}(k)
$$

$\mathbf{w}(k) \sim N\left(0, \mathbf{Q}_{k}\right)$ is the process noise. If the process is assumed to be deterministic $\left(\mathbf{Q}_{k}=0 \forall k\right)$ then by expanding $\mathbf{F}($.) as a Taylor series about the previous prediction, $\hat{\mathbf{x}}(k \mid k)$, and neglecting higher order perturbation terms, we obtain the linearized process model ,

$$
\delta \mathbf{x}(k+1) \approx \mathbf{A} \delta \mathbf{x}(k)
$$

$\delta \mathbf{x}(k+1)=\mathbf{x}(k+1)-\mathbf{x}(k)$

$$
\begin{aligned}
& \mathbf{A}_{[i, j]}=\left[\frac{\left.\partial \mathbf{F}_{[i]}(\mathbf{x}(k))\right]_{\mathbf{x} \hat{\mathbf{x}}(k \mid k)}}{\partial \mathbf{x}_{[j]}}\right. \\
& \mathbf{A}=\left[\begin{array}{cc}
\frac{2 \hat{x}_{2}^{2}(k) \cos \gamma}{\left(2 \hat{x}_{2}(k) \cos \gamma-\hat{x}_{1}(k)\right)^{2}} & \frac{-\hat{x}_{1}^{2}(k)}{\left(2 \hat{x}_{2}(k) \cos \gamma-\hat{x}_{1}(k)\right)^{2}} \\
1 & 0
\end{array}\right]
\end{aligned}
$$

A is the Jacobian matrix of partial derivatives of $\mathbf{F}$ (.) with respect to $\mathbf{x}$. Matrix $\mathbf{A}$ is computed at every new pseudo time step $k$ along the trajectory.

The linear range measurement equation is,

$$
\begin{aligned}
z(k) & =d_{i+1}=\left[\begin{array}{ll}
1 & 0
\end{array}\right]\left[\begin{array}{l}
x_{1}(k) \\
x_{2}(k)
\end{array}\right]+v(k) \\
& =\mathbf{C x}(k)+v(k)
\end{aligned}
$$

where, $\mathbf{C}$ is the observation matrix, and $v(k)$ is the measurement noise assumed $N\left(0, \sigma_{r}^{2}\right)$ and 
uncorrelated. Now, in a manner analogous to [9], the Extended Kalman Filter (EKF) algorithm is used to detect the road edge points.

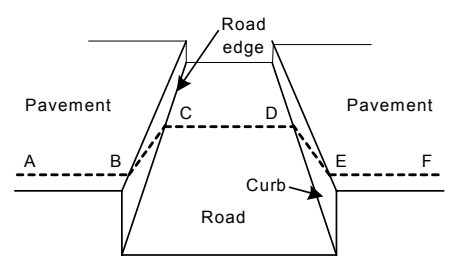

Figure 2: Ideal model of road, curbs and pavements

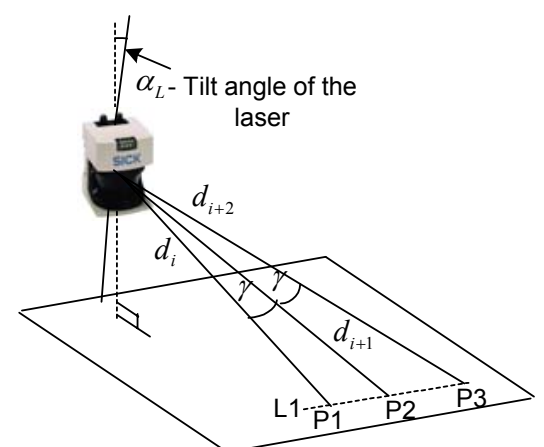

Figure 3: Three consecutive laser data points on a flat road surface

(a) Filter Initialization: A reasonable initial value for the posteriori error covariance, $\mathbf{P}(k \mid k)$ can be estimated using two range measurements, at time $k=1$ and $k=2$. From equation (7), we have $\hat{x}_{1}(1 \mid 1)=z(1)$, $\hat{x}_{1}(2 \mid 2)=z(2)$. From equation (3), we have $\hat{x}_{2}(2 \mid 2)=\hat{x}_{1}(1 \mid 1)$ and hence,

$$
\hat{\mathbf{x}}(2 \mid 2)=\left[\begin{array}{c}
z(2) \\
z(1)
\end{array}\right]
$$

Since the measurement noise is assumed uncorrelated,

$$
\mathbf{P}(2 \mid 2)=\left[\begin{array}{cc}
\sigma_{r}^{2} & 0 \\
0 & \sigma_{r}^{2}
\end{array}\right]
$$

(b) Filter Prediction: computing the predicted measurement and the error covariance matrix,

$$
\begin{aligned}
& \mathbf{A}_{[i, j]}=\frac{\partial \mathbf{F}_{[i]}}{\partial \mathbf{x}_{[j]}}(\hat{\mathbf{x}}(k \mid k)) \\
& \hat{\mathbf{x}}(k+1 \mid k)=\mathbf{F}(\hat{\mathbf{x}}(k \mid k)) \\
& \mathbf{P}(k+1 \mid k)=\mathbf{A} \mathbf{P}(k \mid k) \mathbf{A}^{T}
\end{aligned}
$$

where, $\mathbf{P}(k+1 \mid k)$ is the a priori error covariance matrix, i.e. the state error covariance at time $k+1$, given all observations up to and including time $k$.

(c) Innovation : computing the difference between the observation and the predicted measurement,

$$
\omega(k+1)=z(k+1)-\mathbf{C} \hat{\mathbf{x}}(k+1 \mid k)
$$

(d) Variance of Innovation : the variance associated with the innovation,

$$
s(k+1)=\sigma_{r}^{2}+\mathbf{C P}(k+1 \mid k) \mathbf{C}^{T}
$$

(e) Validation gate :

$$
D(k+1)=\omega^{2}(k+1) s^{-1}(k+1)
$$

An observation that falls outside of a threshold value for the validation, $D(k+1)$ is classified as a possible edge point, i.e. transition from one region to another (e.g. pavement surface to vertical curb surface) and reinitialize the algorithm.

(f) Kalman Filter Gain $\bar{K}(k+1)$ :

$$
\bar{K}(k+1)=\mathbf{P}(k+1 \mid k) \mathbf{C}^{T} s^{-1}(k+1)
$$

(g) State Vector Update and the posteriori state error covariance:

$$
\begin{aligned}
\hat{\mathbf{x}}(k+1 \mid k+1)= & \hat{\mathbf{x}}(k+1 \mid k)+\bar{K}(k+1) \omega(k+1) \\
\mathbf{P}(k+1 \mid k+1)= & \mathbf{P}(k+1 \mid k) \\
& -\bar{K}(k+1) s(k+1) \bar{K}^{T}(k+1)
\end{aligned}
$$

Steps (b) to (g) are repeated for all points of interest along the trajectory.

\subsection{Segmentation results}

In order to simplify subsequent detection of road boundaries, the segmentation is carried out by firstly dividing the range data of a single scan into a left data set and a right data set corresponding to the laser range data to the left and right of the vehicle. Now we apply the above segmentation algorithm to the data points in the left and right data sets to yield point $\operatorname{sets}\left(S_{j}^{l} ; j=1, \ldots n_{1}^{l}\right)$, and $\left(S_{j}^{r} ; j=1, \ldots n_{1}^{r}\right) \quad$ of line segments to the left and right of the vehicle. If it is assumed that the vehicle is more or less moving parallel to the road edges (curbs) and the road edges are locally straight (curvature is small), it can be deduced that the segmented edge point data of the left and the right curbs will be in the left and right point sets 
respectively. Figure 4 shows the laser range data of a single scan and the results of filtering and edge segmentation. The plot symbol ' $x$ ' denotes the actual range data, '*' denotes the predicted data and 'o' denotes a point of discontinuity (an end point of a line segment).

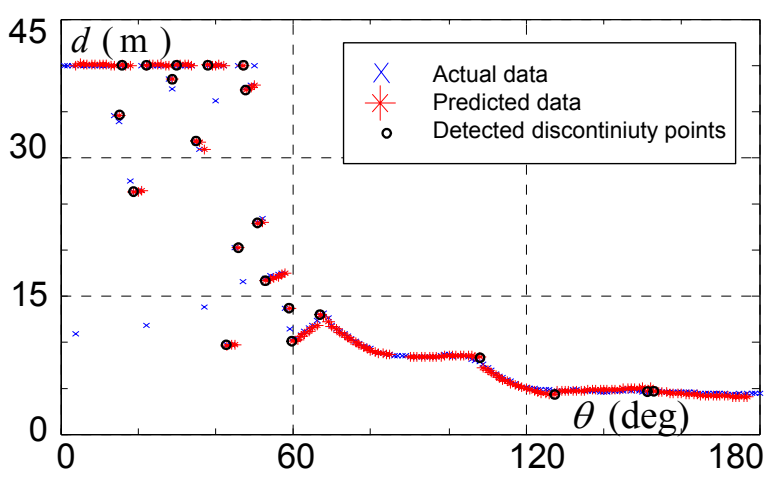

Figure 4: EKF edge segmentation

\section{Feature extraction}

In this section, we use the method of least squares to fit straight lines to the edge data sets determined in section 2. The computed lines are used to extract the straight lines corresponding to the left and right curbs, which are assumed locally straight.

\subsection{Fitting lines to edge segments}

Let us denote $d(P, l)$ as the distance of a point $P$ from a line $l$. Our objective is to fit a line given a set of points $P_{\alpha}=\left(a_{\alpha}, b_{\alpha}\right), \alpha=1, \ldots . N$, such that the sum of the squares of the errors, $e$, is minimized, i.e.

$$
e=\sum_{\alpha=1}^{\mathrm{N}} d\left(P_{\alpha}, l\right)^{2} \rightarrow \min
$$

Let the line to be fitted is expressed in the Hesse normal form,

$$
x \cos \theta+y \sin \theta=\rho, \quad \rho \geq 0
$$

It can be shown that the solution is given by,

$$
\begin{aligned}
& \theta=\frac{1}{2} \tan ^{-1} \frac{2 \sum_{\alpha=1}^{N} a_{\alpha}^{\prime} b_{\alpha}^{\prime}}{\sum_{\alpha=1}^{N}\left(a_{\alpha}^{\prime}\right)^{2}-\sum_{\alpha=1}^{N}\left(b_{\alpha}^{\prime}\right)^{2}}+\frac{n \pi}{2}, \\
& \rho=\bar{a} \cos \theta+\bar{b} \sin \theta
\end{aligned}
$$

where,

$$
\begin{aligned}
& \bar{a}=\frac{1}{N} \sum_{\alpha=1}^{N} a_{\alpha}, \quad \bar{b}=\frac{1}{N} \sum_{\alpha=1}^{N} b_{\alpha}, \\
& a_{\alpha}^{\prime}=a_{\alpha}-\bar{a}, \quad b_{\alpha}^{\prime}=b_{\alpha}-\bar{b},
\end{aligned}
$$

and $n$ is an arbitrary integer. There are four solutions that exist for $\theta$ in the interval $0 \leq \theta \leq 2 \pi$, among which one solution is chosen such that,

$$
\begin{aligned}
& \bar{a} \cos \theta+\bar{b} \sin \theta \geq 0 \\
& \text { If } \sum_{\alpha=1}^{N} a_{\alpha}^{\prime} b_{\alpha}^{\prime}>0 \text { then } \frac{\pi}{2}<\theta<\pi, \quad \frac{3 \pi}{2}<\theta<2 \pi \\
& \text { If } \sum_{\alpha=1}^{N} a_{\alpha}^{\prime} b_{\alpha}^{\prime} \leq 0 \text { then } 0 \leq \theta \leq \frac{\pi}{2}, \quad \pi \leq \theta \leq \frac{3 \pi}{2}
\end{aligned}
$$

Now we choose only those lines whose residual error, $e$, is smaller than a given threshold. This is mainly because the lines to be extracted are those corresponding to the curbs, which are locally straight and also to filter out lines fitted to noisy and spurious data. Suppose the left and right sets of lines so determined are:

$$
\begin{aligned}
& L_{1}^{l}=\left\{\left(\rho_{i}^{l}, \theta_{i}^{l}, e_{i}^{l}\right), i=1 \cdots n_{1}^{l}\right\} \\
& L_{1}^{r}=\left\{\left(\rho_{i}^{r}, \theta_{i}^{r}, e_{i}^{r}\right), i=1 \cdots n_{1}^{r}\right\}
\end{aligned}
$$

where, $e_{i}^{l}$ is the minimum error in fitting the $i^{\text {th }}$ set of left edge points $S_{i}^{l}$. Similarly, for $e_{i}^{r}$. The filtered data (in Cartesian coordinates) corresponding to the set of lines, $L_{1}^{l}$ and $L_{1}^{r}$ are shown in Figure 5.

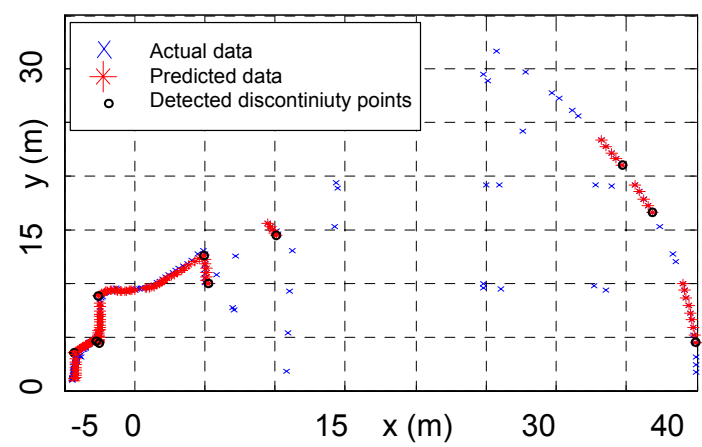

Figure 5: Laser data in Cartesian coordinates

\subsection{Road curb detection}

In the event both left and right curbs exists, the line segments corresponding to the curbs is extracted as follows. Firstly, we determine the line segments whose orientations are the same as the orientation, $\theta_{v}$ of the vehicle with respect to the straight curbs to within a given tolerance, $\delta \theta$ in each of the two sets of lines. 


$$
\begin{aligned}
& L_{2}^{l}=\left\{\left(\rho_{i}^{l}, \theta_{i}^{l}, e_{i}^{l}\right),\left|\theta_{v}-\theta_{i}^{l}\right| \leq \delta \theta, i \in\left(1, \cdots n_{2}^{l}\right)\right\} \\
& L_{2}^{r}=\left\{\left(\rho_{i}^{r}, \theta_{i}^{r}, e_{i}^{r}\right),\left|\theta_{v}-\theta_{i}^{r}\right| \leq \delta \theta, i \in\left(1, \cdots n_{2}^{r}\right)\right\}
\end{aligned}
$$

Now, from the two sets of lines parallel to the curbs given in (20), choose a pair of lines (one line from the left $L_{2}^{l}$ and one from the right $L_{2}^{r}$ ) such that their interline distance is closest to the road width. That is to say we select the pair of lines that are parallel to the curbs and their interline distance is approximately the width of the road. In case, there are several such pairs, the pair corresponding to the minimum lateral distance form the vehicle coordinate system is chosen.

The results of the road curb edge lines extracted for a single scan of laser range data (Figure 4), are as shown in Figure 6. Figure 7 shows the curb lines extracted in a rainy environment. It is to be noted that the specular reflections due to puddle of water in the middle of road does not alter curb extraction accuracy. Figure 8 shows the effectiveness of the algorithm in night driving.

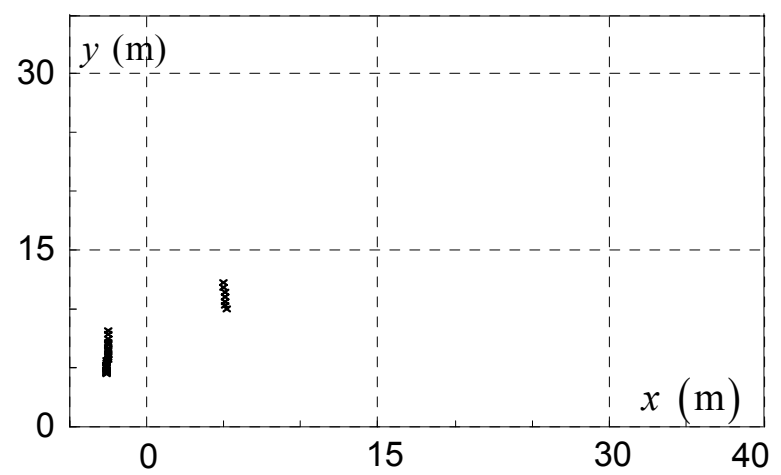

Figure 6: Extracted road boundaries

\subsection{Intersection detection}

In the very first range scan, we assume a priory knowledge about the vehicle orientation with respect to road boundaries $\left(\theta_{v}\right)$, road width $(w)$ and line parameter $(\rho)$. For consequent scans, bounds for the $\theta_{v}$ is chosen using the estimated orientation with respect to the vehicle in the previous sampling time, road curvature ahead and change of the absolute orientation of the vehicle. Mean value of the estimated road widths in the previous time stamps is used as the current road width. Parameter $\rho$ in the previous scan is also used.

Now we consider a situation where the vehicle approaches a road intersection (Figure 9). In the left hand side of the road, it could be seen a continuous pavement curb (" $A B$ "), while on the right hand side, there is no pavement curb in segment " $D E$ " as it is a road intersection. When the range data happens to be fallen on to the section " $D E$ " in Figure 9, there are no line sets which can be extracted as road curbs using the road width information. In this kind of situations, we use the evolution of $(\rho, \theta)$ with time in order to correctly filter out non-existence edges while keeping the existing one-sided edges (Figure 13).
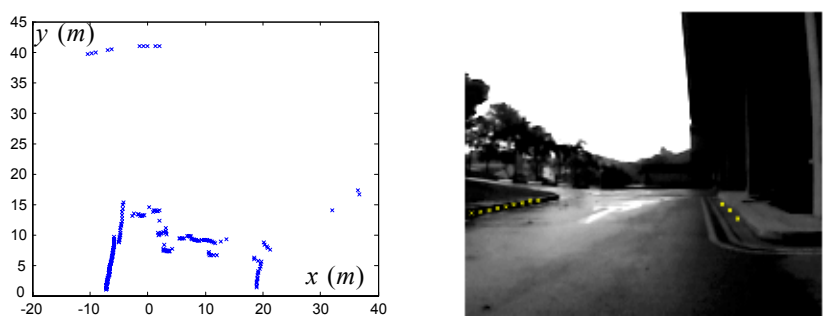

Figure 7: Rainy environment (note the specular reflections due to puddle of water in the middle of road).
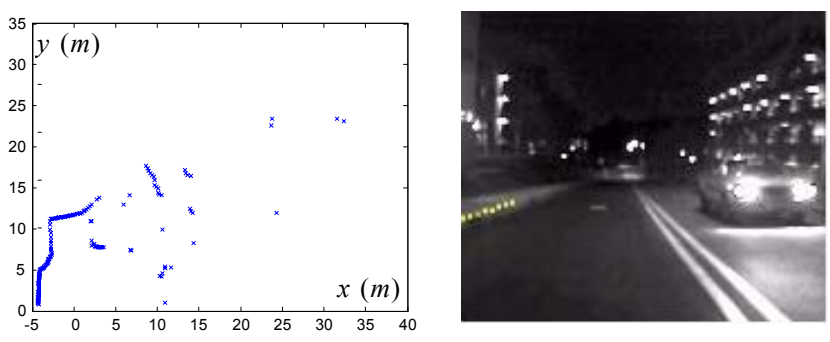

Figure 8: In the night with another vehicle

Raw laser data of a drive on such an environment is shown in Figure 10. The estimated orientation with respect to the road of the previous time stamp is shown in Figure 11. Estimated road width of the previous time stamp is shown in Figure 12. Most of the time the estimated road width is around $7.5 \mathrm{~m}$ and in some places it rises to about $20-30 \mathrm{~m}$ demonstrating that it may not be road boundaries. The algorithm is capable of handling "Y-junctions" as shown in Figure 13.

\section{Conclusions}

Sensing and detection of road boundaries and intersections in real time are important tasks in the synthesis of autonomous vehicles and driver assistance systems. In this respect, camera based vision sensors and associated techniques have proved to be useful and effective as reported in the literature. However, unfavorable lighting conditions (overcasts, sun's glare, bad weather, and shadows) and other effects (blurring of images due to vibrations of the moving vehicle) can impair the vision image quality and render the performance of vision based systems unusable unless sophisticated image processing techniques are used at the expense of processing speed. To overcome the problems of vision sensing and processing techniques, the paper proposes an alternative methodology using a 2D laser range measuring system. Laser depth measurements provide for speedy extraction of two 
corresponding road edges or curbs using the laser depth measurements and an Extended Kalman filter (Road curbs unlike painted lane markings are not conspicuous and hence not easily extracted from a camera image). It is also shown using experiments that the proposed system is capable of detecting Y-intersections. Our future work will include the generalization of the algorithm for handling "T-junctions" and " $\mathrm{x}$ intersections".

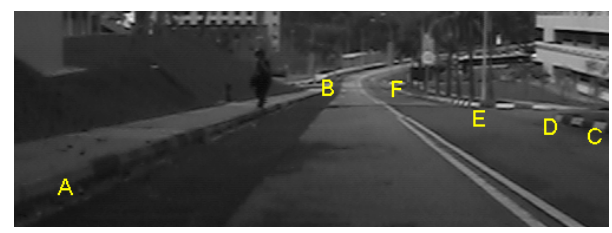

Figure 9: Experimental site

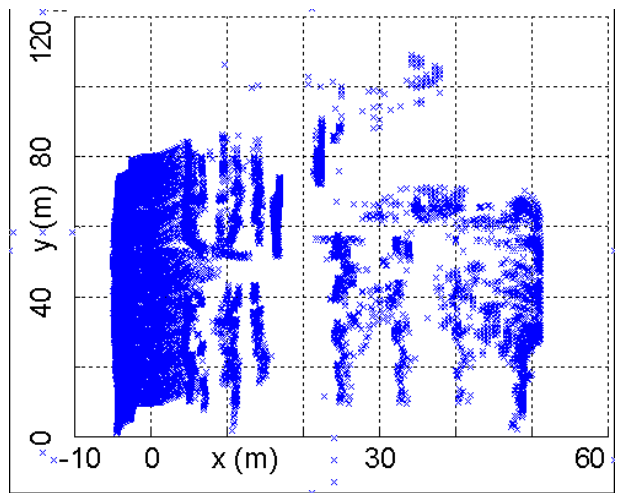

Figure 10: Raw data

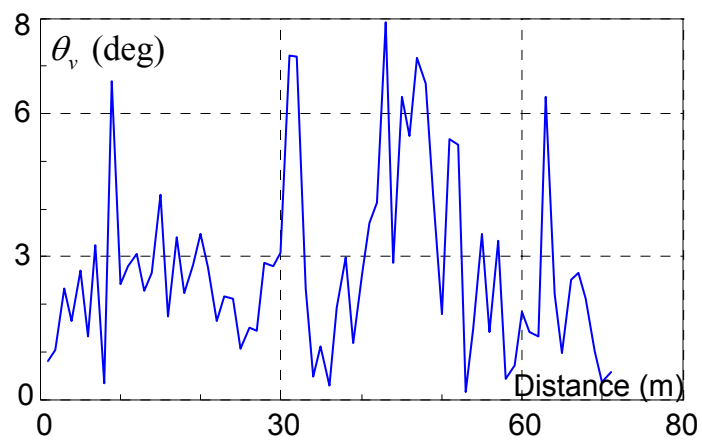

Figure 11: Estimated vehicle orientation

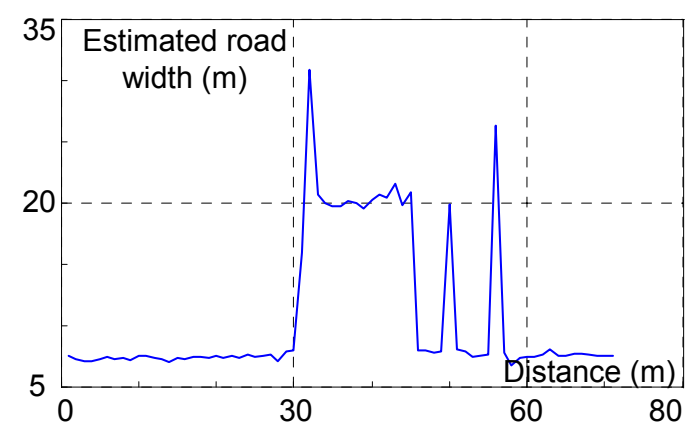

Figure 12: Estimated road width

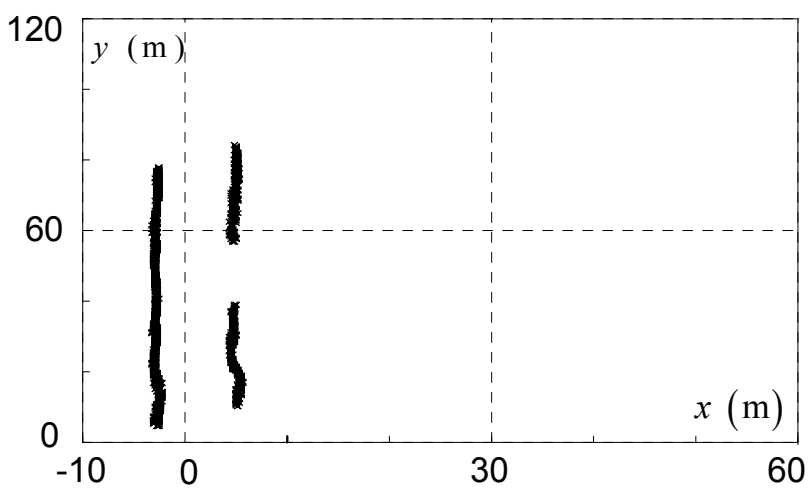

Figure 13: Road boundary extraction

\section{References}

[1] D. Pomerleau and T. Jochem, "Rapidly Adapting Machine Vision for Autonomated Vehicle Steering", IEEE Expert, Vol.11 Issue: 2 , pp.1927, 1996.

[2] M. Bertozzi and A. Broggi, "GOLD: A Parallel Real-Time Stereo Vision System for Generic Obstacle and Lane Detection", IEEE Transactions on Image Processing, Vol.7, No.1, pp.62-81, Jan. 1998.

[3] K. Kaliyaperumal, S. Lakshmanan and K. Kluge, "An Algorithm for Detecting Roads and Obstacles in Radar Images", IEEE Transactions on Vehicular Technology, Vol.50, No.1,pp.170-182, Jan. 2001.

[4] T. Dunlay, "Obstacle Avoidance Perception Processing for the Autonomous Land Vehicle", Proceedings of the Conference of IEEE Robotics and Automation, Vol.2, 1998, pp. 912-917.

[5] Technical Description, SICK AG, Auto Ident, Germany.

[6] L. Henriksen and E. Krotkov, "Natural Terrain Hazard Detection with a Laser Rangefinder", Proceedings of the IEEE International Conference on Robotics and Automation, New Mexico, 1997, pp.968-973.

[7] D. Dedieu, V. Cadenat and P. Soueres, "Mixed cameralaser based control for mobile robot navigation", Proceedings of IEEE/RSJ International Conference on Intelligent Robots and Systems, 2000.

[8] J. A. Castellanos, J. Neira and D. Tardos, "Multisensor Fusion for Simultaneous Localization and Map Building", IEEE Transactions on Robotics and Automation, Vol.17, No.6, pp.908914, 2001.

[9] M. D. Adams, Sensor Modelling, Design and data processing for Autonomous Navigation, World Scientific Publishing Co. Pte. Ltd, 1999. 